\title{
Advancing Clinical and Transformational Research with Informatics at the University of Kansas Medical Center
}

\author{
Lemuel Russell Waitman', Gerald Lushington², Judith J. Warren ${ }^{3}$
}

B iomedical Informatics accelerates scientific discovery and improves patient care by converting data into actionable information. Pharmacologists and biologists receive improved molecular signatures; translational scientists use tools to determine potential study cohorts; providers view therapeutic risk models individualized to their patient; and policy makers can understand the populations they serve. Informatics methods also lower communication barriers by standardizing terminology describing observations, integrating decision support into clinical systems, and connecting patients with providers through telemedicine.

The University of Kansas Medical Center's (KUMC) decision to pursue a National Institutes of Health (NIH) Clinical and Translational Science Award $(\mathrm{CTSA})^{1}$ in partnership with other regional academic medical centers, universities, and health systems catalyzed the development and integration of informatics capabilities to specifically support translational research in our region and to address issues and needs like those identified above. Headquartered at KUMC, the NIH-CTSA-supported Frontiers program, also called The Heartland Institute for Clinical and Translational Research (HICTR), is a network of scientists from institutions in Kansas and the Kansas City region (www.FrontiersResearch.org). The vision for the informatics section is to provide rich information resources, services, and communication technologies across the spectrum of translational research.
Broadly the initiative would: a) adopt methods which facilitate collaboration and communication both locally and nationally, b) convert clinical systems into information collection systems for translational research, c) provide innovative and robust informatics drug and biomarker discovery techniques, $\mathrm{d}$ ) work with state and regional agencies to provide infrastructure and data management for translational outcomes research in underserved populations, and e) measure clinical information systems' ability to incorporate translational research findings.

The specific aims for informatics target translational needs requiring further investment and complement the novel methods and technologies of our region: a) the Institute for Advancing Medical Innovation's (IAMI) ${ }^{2}$ effort in drug discovery and b) the Personalized Medicine Outcomes Center at the Uni-

1 Director Medical Informatics, Department of Biostatistics, University of Kansas Medical Center

2 Associate Scientist and Director of Laboratories, Molecular Structures Group, University of Kansas

3 Christine A. Hartley Centennial Professor, School of Nursing, University of Kansas Medical Center 
versity of Missouri- Kansas City (UMKC) which seeks to understand the determinants of health systems' outcomes and develop methods to deliver evidence based practice. Four aims were articulated:

1. Provide a portal for investigators to access clinical and translational research resources, track usage and outcomes, and provide informatics consultation services.

2. Create a platform, HERON (Healthcare Enterprise Repository for Ontological Narration), to integrate clinical and biomedical data for translational research.

3. Advance medical innovation by linking biological tissues to clinical phenotype and the pharmacokinetic and pharmacodynamic data generated by research in phase I and II clinical trials (addressing T1 translational research).

4. Leverage an active, engaged statewide telemedicine and Health Information Exchange (HIE) to enable community based translational research (addressing T2 translational research).

This article will focus on Frontiers' plan and progress in achieving these aims since the grant was awarded in June 2011.

\section{Background}

The University of Kansas has a wide range of informatics capabilities, developed over decades of research and service. Through the National Center for Research Resources (NCRR) IDeA Networks for Biomedical Research Excellence (INBRE), the Kansas' KINBRE has created a ten campus network of collaborative scientists using common bioinformatics resources. Kansas has pio- neered the use of telemedicine since the early 1990s, providing inter-state connectivity at over 100 sites and conducting thousands of clinical consultations and hundreds of educational events for health care professionals, researchers, and educators.

The Center for Health Informatics' Simulated E-hEalth Delivery System, a jointly funded program between the University of Kansas and Cerner Corporation, creates an equitable partnership to fully integrate applied clinical informatics into an academic setting and supports over 40 international academic clients. These existing efforts are complemented by more recent investments in clinical research informatics and medical informatics.

Starting in 2005, KU selected Velos e-Research for our Clinical Research Information System (CRIS). CRIS is a web application, supports Health Level 7 messaging for systems integration, and complies with industry and federal standards (CFR Part 11) for FDA regulated drug trials. It consists of a management component for patients, case report forms, and protocols, and allows financial management for conducting studies with administrative dashboards and milestones for measuring research effectiveness. KU's support and staffing serves not only support KU investigators but also to provide enterprise licensing for Frontiers' investigators to conduct multi-center, cooperative group, and investigator-initiated research with CRIS across unlimited participating locations without additional license fees. CRIS has been deployed throughout our institution and the team has experience sup- 
porting multicenter trials across the nation.

Bioinformatics is a critical technology for translational (T1) research that systematically extracts relevant information from sophisticated molecular interrogation technologies. Analytical techniques-such as microarray experiments, proteomic analysis and high throughput chemical biology screeningcan probe disease etiology, aid in development of accurate diagnostic and prognostic measures, and serve as a basis for discovering and validating novel therapeutics. HICTR institutions benefit from strong molecular bioinformatics research expertise distributed across three synergistic entities: the Center for Bioinformatics at KU-Lawrence (CBi), the Bioinformatics and Computational Life Sciences Research Laboratory (BCLSL), and the K-INBRE (Kansas IDeA Network for Biomedical Research Excellence) Bioinformatics Core (KBC). Over time, CBi (an internationally recognized structural biology modeling research program, focusing on computational structural biology) and BCLSL (a research program, led by the School of Engineering at KU-Lawrence and focusing on development of sophisticated algorithms for mining biological data) should grow into collaborative partners for Frontiers investigators.

The University of Kansas Center for Health Informatics (KU-CHI), established in 2003, is an interdisciplinary center of excellence designed to advance health informatics through knowledge integration, research, and education of faculty and students in the expanding field of biomedical science and infor- mation technology. Like many funded CTSA programs, KU is a founding member of the American Medical Informatics Association's (AMIA) Academic Forum. Graduate health informatics education, continuing education, AMIA 10x10 program, consultation, and staff development workshops/seminars designed to advance health informatics are sponsored or co-sponsored through KU-CHI. Graduate education in health informatics began in 2003 in the School of Nursing and is now a multidisciplinary master's degree program offered by the Office of Graduate Studies and managed by the KU-CHI. Helen Connors, $\mathrm{PhD}, \mathrm{RN}, \mathrm{FAAN}$ (executive director of KU-CHI) chairs the State of Kansas EHealth Advisory Council that oversees the development of the state's health information exchange strategic and operational plans and is a board member of the Kansas City Bi-state Health Information Exchange, the metropolitan area's Regional Health Information Organization.

Under the direction of Dr. Ryan Spaulding, The University of Kansas Center for Telemedicine \& Telehealth (KUCTT) is a leader in telehealth services and research. The program began in 1991 with a single connection to a community in western Kansas. The Kansas telehealth network now is used to connect 60 health facilities in Kansas each year. It also is the basis for several national and international collaborations, demonstrating significant potential of telehealth technologies to eliminate distance barriers. Over the last 19 years, nearly 30,000 clinical consultations have been conducted across nu- 
merous allied health, nursing and medical specialties, making the KUCTT one of the earliest and most successful telehealth programs in the world. In 2009, over 5000 Kansas patients benefited from telemedicine services that included clinical consultations, community education, health screenings and continuing education for health professionals. Through the Midwest Cancer Alliance (MCA) alone, the KUCTT supported numerous second opinion cancer consultations, multidisciplinary tumor board conferences, chemotherapy administration courses and routine patient consultations. Other specialties facilitated by the KUCTT include cardiology, psychology, psychiatry, neurology, wound care, etc. The KUCTT also has been very active integrating numerous technologies to support clinical and research activities, including telehealth systems, electronic health records, data registries and patient management systems.

In 2010, KU committed \$3.5 million dollars over the next five years towards medical informatics academics and service, and established the Division of Medical Informatics in the Department of Biostatistics, to integrate clinical research informatics, and to provide overall leadership for the Frontiers Biomedical Informatics program. Coincidental with establishing the division, the University of Kansas Hospital completed their five-year, \$50 million investment to implement the Epic Electronic Medical Record, concluding with Computerized Provider Order Entry in November 2010. This effort has transformed the inpatient environment. Subsequently, the Univer- sity of Kansas Physicians began adoption of Epic across all ambulatory clinics.

Planned Program Objectives and Progress.

Aim \#1: Provide a Frontiers portal for investigators to access clinical and translational research resources and provide informatics consultative services.

As shown at the top of Figure 1, a web application to link research resources and foster communication was seen as an underlying need. It provides investigators access to request services from the Translational Technologies and Resource Center, the Participant \& Clinical Interactions Resources, the Community Partnership for Health, and the Ethics section. It also allows investigators to obtain targeted internal and extramural funding opportunities, request biostatistics support and clinical research database construction, request and access informatics resources, and explore educational offerings.

A key component of the portal is the adoption of REDCap ${ }^{4}$ (Research Electronic Data Capture), to create databases to manage requests and triage review and tracking of requests. Medical Informatics implemented REDCap in January 2011 to provide a self-service database management system for clinical and translational research. Since users become familiar with the ease of use and development of forms, it was a natural approach to also extend the use of REDCap to manage research request activities. Thus, the user focused portal is complemented by using REDCap to help Frontiers management track projects, distribute resources, and facilitate evalu- 
ation similar to efforts at Vanderbilt University $^{3}$. Frontiers adoption of REDCap has been dramatic with over 700 users of over 500 projects either under development or in production. REDCap usage has ranged from student research projects as part of a fourth year Health of the Public medical school course to providing the data management infrastructure for a $\$ 6.3$ million NIH funded Alzheimer's Disease Core Center grant. In addition to the existing capabilities of the CRIS, there are several informatics specific components have been developed to provide better integration for clinical and translational researchers. Integrated authentication between the portal, the campus Central Authentication Service, CRIS, and i2b2 streamlines process for submitting data requests and receiving data and complementary methods to support Data Request Oversight Committee activities. Finally, we provided a request management informatics consult service and training described below.

With the award of the CTSA, we anticipated the need to dedicate a clinical informaticist to match investigators' needs against current information available in Frontiers resources, specifically the HERON repository described in Aim 2. By early 2012, we had recruited Tamara McMahon to serve in this role as the clinical informatics coordinator. She assists users with tools like i2b2 and will recommend alternative approaches when required data are not integrated.

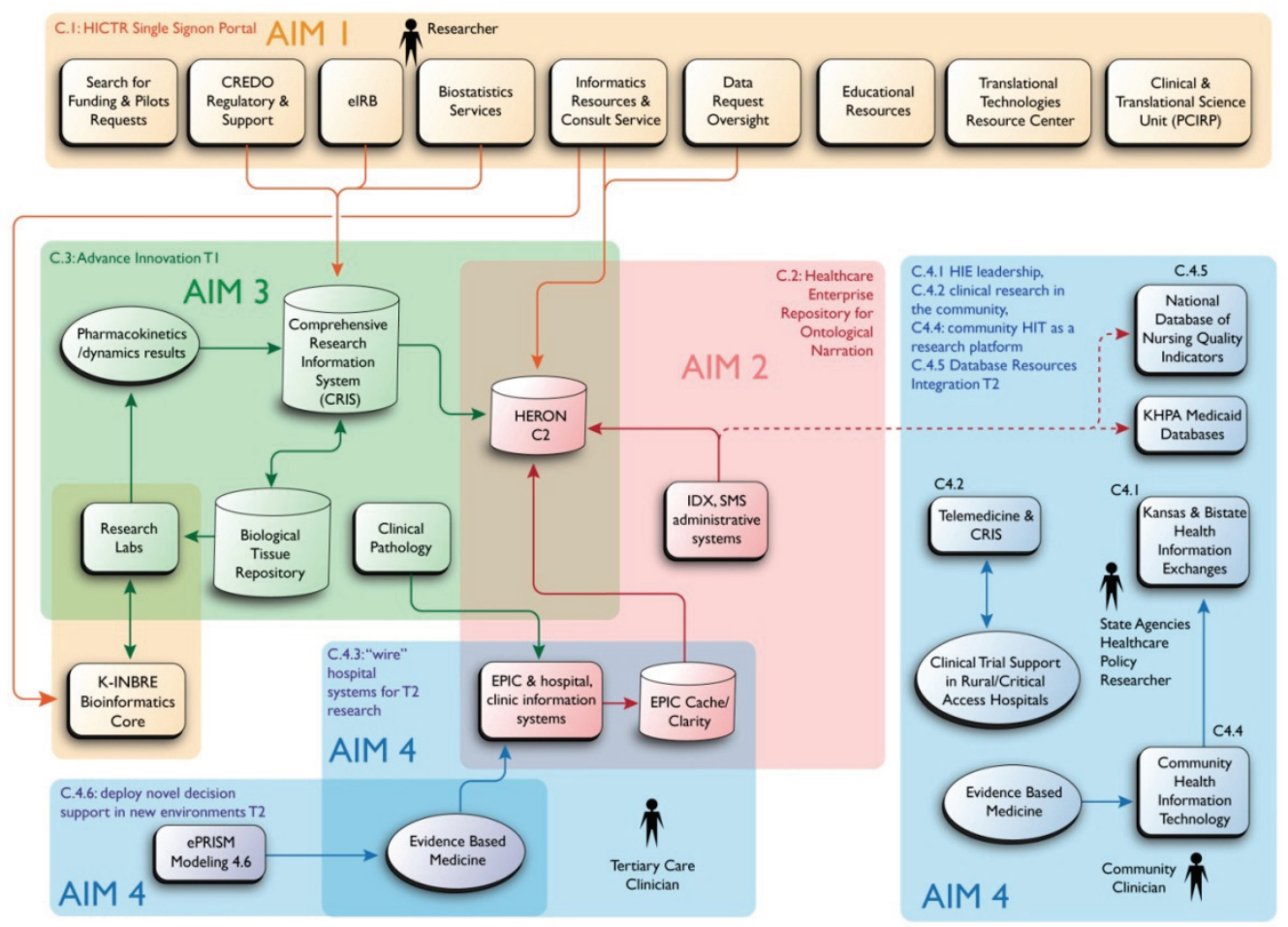

Figure 1. Conceptual Model of Biomedical Informatics and Specific Aims

access to our clinical data repository described in the second aim. REDCap was used to develop secure methods for the
She and the larger team's experience from front-line consulting with translational researchers drives prioritizing ad- 
ditional data sources, terminology and ontology development and guides the adoption of new technologies for data retrieval and analysis. As we've matured as a team these consultative services, while lead by the coordinator, are staffed and addressed by the combined biomedical informatics personnel. Additionally, informatics clinics have been established biweekly for investigators, staff, and students to attend and discuss needs and solutions in an informal group setting. Requests for bioinformatics and computational biology capabilities are facilitated by the Bioinformatics Resources section of the portal and support by the bioinformatics assistant director (initially Dr. Gerald Lushington; currently Dr. Paul Terranova).

Aim \#2: Create a platform, HERON (Healthcare Enterprise Repository for Ontological Narration), to integrate clinical and biomedical data for translational research.

Transforming observational data into information and knowledge is the cornerstone of informatics and research. The opportunity for data driven knowledge discovery has exploded as health care organizations embrace clinical information systems. However, when dealing with confidential patient information, science and technology are dependent on law, regulation, organizational relationships, and trust. HERON provides Frontiers with secure methods for incorporating clinical data into standardized information aligned with national research objectives. It facilitates hypothesis generation, allows assessing clinical trial enrollment potential, and minimizes duplicate data entry in clini- cal trial data capture, outcomes research, and evaluation of $\mathrm{T} 2$ interventions for translating research into practice. While initially focused on integrating data between the KU Medical Center, the KU Hospital, and KU affiliated clinics, our methods are designed to subsequently integrate information among all Frontiers network institutions.

Beginning in April 2009, the Frontiers started piloting a participant registry in the clinics which allows patients to quickly consent to be contacted by clinical researchers. The registry contains the consented patients' demographics, contact information, diagnoses, and procedure codes. From April to July 2010, the medical center, hospital and clinics reviewed comparable practices ${ }^{5,6,7,8}$ and drafted a master data sharing agreement between the three organizations that was signed September 6, 2010. The HERON executive committee is composed of senior leadership (e.g. chief operating, financial, executive officers and chief of staff) from the hospital, clinic and medical center and provides governance for institutional data sharing. Establishing business processes and servicing research requests is conducted by the Data Request Oversight Committee (DROC) which reports to the HERON executive committee. The repository's construction, oversight process, system access agreement, and data use agreement for investigators were approved by the Institutional Review Board. Since HERON is currently funded by $\mathrm{KU}$ and contains medical center and affiliated clinics and hospital data, access requires a medical center investigator. As additional institutions and health care organizations pro- 
vide support and contribute data, they will be incorporated with multiinstitutional oversight provided by the HERON executive committee and DROC.

As agreed upon by the HERON executive committee, HERON has four uses: cohort identification, de-identified data, identified data, and participant contact information:

After signing a system access agreement, cohort identification queries and view-only access is allowed and activity logs are audited by the DROC.

Requests for de-identified patient data, while not human subjects research, are reviewed by the DROC.

Identified data requests require approval by the Institutional Review Board prior to DROC review. After both approvals, medical informatics staff will generate the data set for the investigator.

Investigators who request contact information for cohorts from the HICTR Participant Registry have their study request and contact letters reviewed for overlap with other requests and adherence to policies of the Participant and Clinical Interactions Resources Program Data Request Committee.

The utility of data for clinical research is proportional to the amount of data and the degree to which it is integrated with additional data elements and sources. However, as additional data are integrated and a richer picture of the patient is provided, privacy concerns increase. HERON receives and preserves data into an identified repository, links and transforms those data into deidentified, standardized concepts, and allows users to retrieve data from this separate de-identified repository (See Figure 2). We recognize that certain research requires access to identified data but our approach streamlines oversight when needs are met with de-identified data. We adopted the NIH funded i $2 b 2^{9}$ software for user access, project management, and hypothesis exploration and chose Oracle as our database because of existing site licensing and $i 2 b 2$ compatibility. Transformation and load activities are written in the python language and deployed onto SUSE LINUX servers. Servers are maintained in the medical center and hospital's data center which complies with HIPAA standards for administrative, technical and physical safeguards.

Maximizing data sharing to adhere to NIH guidelines has been a key goal for the development of HERON. Our goals are to make scientific data and informatics methods available as widely as possible while safeguarding the privacy of our patients. Our data sharing agreements were developed at an organizational level to streamline requests for deidentified data. Our repository's use of i2b2 and UMLS terminologies facilitate national collaboration. By using open source development tools and languages all new software developed using support from the NIH grant is then made available to others through our division's research and development website: http://informatics.kumc.edu.

Access to the identified repository is highly restricted and monitored. Data exchange between source systems, HERON, and user access is handled via security socket layer communications, https with digital certificates, and 128-bit 
or higher public key cryptography (ex: $\mathrm{SSH}-2)$. We will continue to monitor federal guidance regarding appropriate security measures for healthcare information $^{10}$. User access, activity logs, and project management for the deidentified repository is managed via the i2b2 framework and is integrated with the medical centers' open source Jasig Central Authentication Service and the HICTR portal. Account creation and access control is provided by the medical center. Audit logs are maintained for both the identified and de-identified repositories. Intrusion detection mechanisms are used and monitored by medical center Information Resources security personnel who also conduct HIPAA Security Rule reviews of the systems.

Current literature highlights challenges with providing anonymity after de-identifying data ${ }^{11,12,13,14}$. While we remove all 18 identifiers to comply with HIPAA Safe Harbor, the de-identified repository is treated as limited data set, reinforced by system access and data use agreements. Initially, HERON will only incorporate structured data. We will stay abreast of best practices (e.g., De-ID, the
MITRE Identification Scrubber Toolkit versus only extracting concepts) prior to incorporating narrative text results which have greater risk for reidentification ${ }^{11,15}$.

Arvinder Choudhary, our medical informatics project director, and Daniel Connolly, our lead biomedical informatics software engineer, were responsible for the initial construction of HERON. Starting in April 2010, the team has deployed a data repository with i2b2 integration, contributed to the $\mathrm{i} 2 \mathrm{~b} 2$ community code for integration with the Central Authentication Service (an open source project used by many universities for "single sign-on"), worked with other CTSA institutions to use the UMLS as a source terminology for $\mathrm{i} 2 \mathrm{~b} 2$, and established a software development environment for the team using Trac and Mercurial. Initial pilot use began as early as April for access to the Frontiers Participant Registry (diagnoses, demographics, and procedures based on clinic billing systems). The current architecture was deployed in August 2010 and populated with production data from IDX and Epic in September 2010.

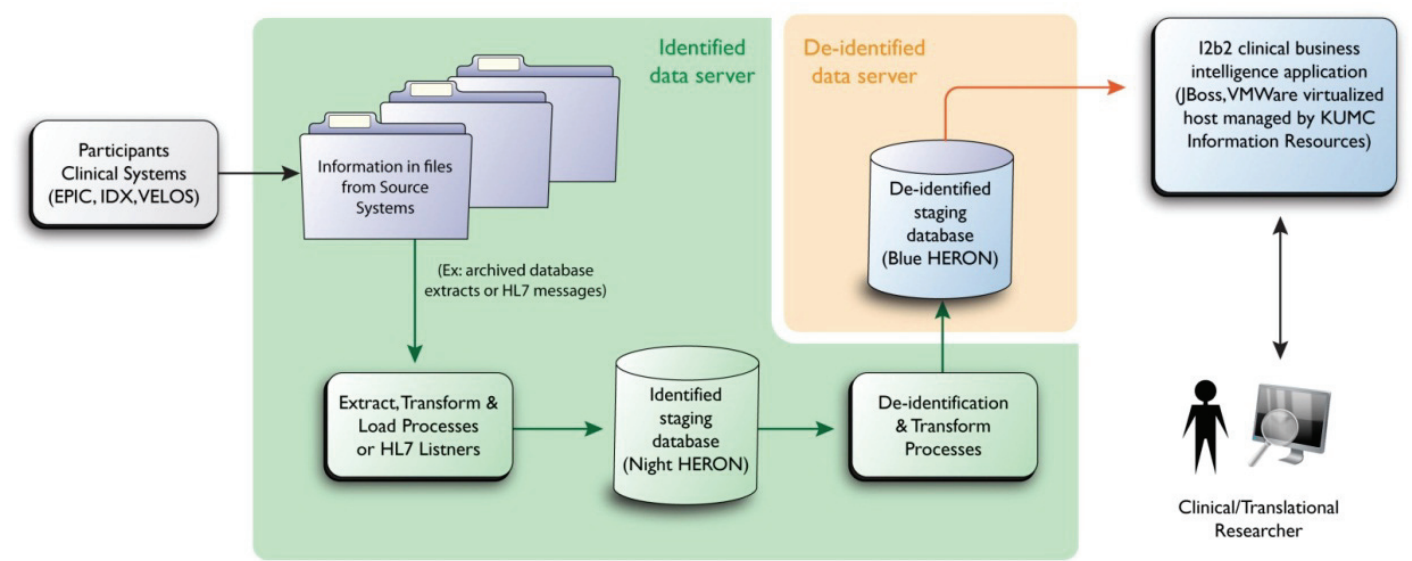

Figure 2. HERON Architecture 
Initial extract, load, and transform processes obtain data files from the source system via secure file transfer methods. The repository was available as a proof of concept in December 2010 and entered beta mode for all faculty in March 2011. HERON has been updated with new data and functionality every month since August 2011 (updates advertised on our blog:

http://informatics.kumc.edu/work/blog). As of August 2012, HERON is using an i2b2 version 1.6 and it contains approximately 850 million facts for 1.9 million patients.

From its inception, HERON has benefitted from $i 2 b 2$ community expertise and the team works with other academic medical centers to align our terminology with CTSA institutions. Our approach is to rely on the National Library of Medicine Unified Medical Language System (UMLS) as the source for ontologies and to use existing or develop new update processes for the i2b2 Ontology Management Cell and other systems. The National Center for Biological Ontologies is also working with the $\mathrm{i} 2 \mathrm{~b} 2$ development team to create methods that can assist organizations using $\mathrm{i} 2 \mathrm{~b} 2$ with building appropriate ontologies based on the UMLS or other source ontologies. This may play a greater role as we bridge clinical data towards bioinformatics domains and to incorporate more recently authored ontologies that are not fully supported by the NLM. As we develop new mapping for going from UMLS into $i 2 b 2$ and from source systems such as Epic, we have shared

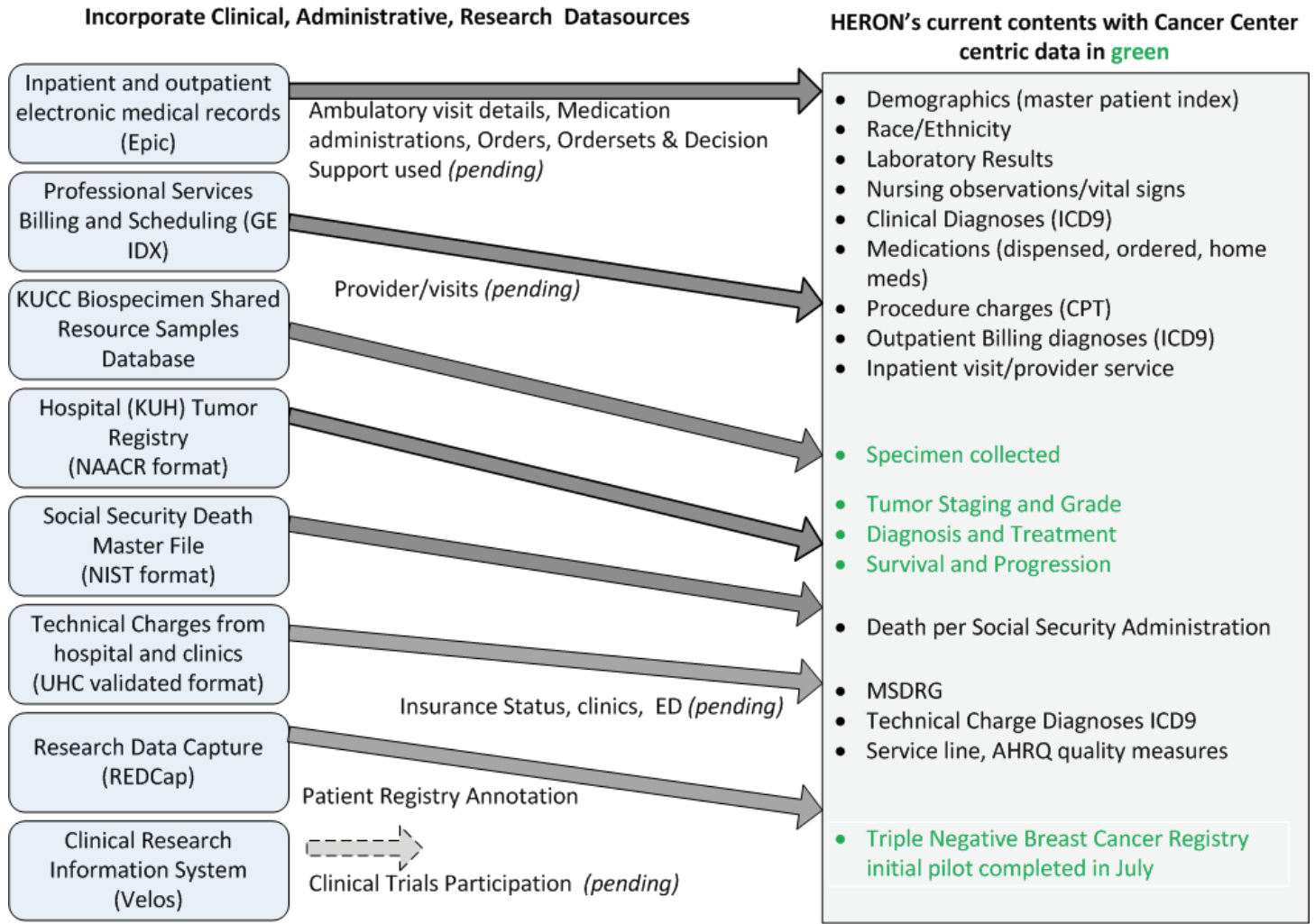

Figure 3. HERON Progress on Incorporating Data Sources and Ontologies

Status as of August 17, 2012 
our code and processes with the i2b2 community via our website. Figure 3 illustrates our current progress in building a rich picture of our patient population and highlights places where the HERON repository also serves to advance KUMC's goal to become a National Cancer Institute designated Cancer Center.

The team's approach leveraged methods outlined by several CTSA organizations that shift mapping inside i2b2 instead of in database transformation and load processes ${ }^{16}$. This preserves the original data side by side with the transformed information allowing easier review by investigators and domain experts. We applied these methods when we incorporated nursing flowsheet information ${ }^{17}$ (vital signs, nursing assessments, social status) from the Epic electronic medical record. Judith Warren, PhD, RN, BC, FAAN, FACMI (Director of Nursing Informatics, the Center for Health Informatics) serves as assistant director of health informatics for Frontiers and has extensive experience developing health informatics standards and terminologies, teaching informatics, and developing national policy for health information technology. She has led several initiatives, especially regarding nursing observations, to adopt standards that will maximize data integration and reuse.

Our hope is that standardizing information for research strengthens our relationship with the hospital and clinics. Reusing information for clinical quality improvement also requires

\section{Frontiers Participant Data Richness Today}

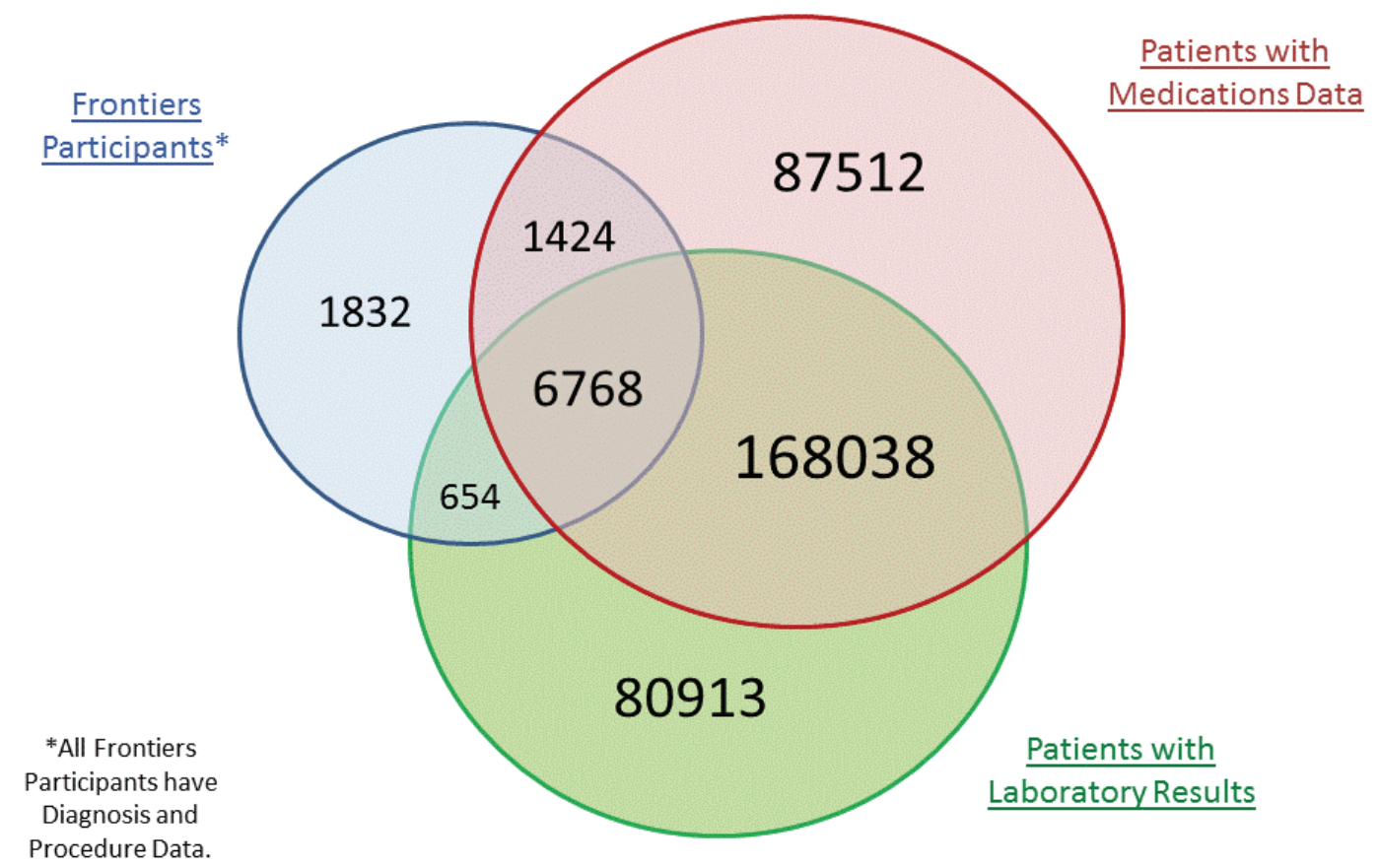

Figure 4. Richness of Phenotype in HERON using Frontier Participants as an example 
standardization. Translational research using HERON has highlighted areas needing terminological clarity and consistency in the electronic medical record. Going forward, we are partnering with Dr. Ator, Chief Medical Information Officer, and the University of Kansas Hospital's Organizational Improvement (which has two of their leaders on the DROC) to develop a data driven process for improving standardization. Research and quality improvement goals also align with achieving "meaningful use"18 and Health Information Exchange activities. We will gradually build relationships by initially focusing on linking data sources from KU Hospital and affiliated clinics. Figure 4 provides an illustration of how today, patients who volunteer to be contacts for research in the clinic based upon a clinic billing system indicator are linked to medication exposure and laboratory results, allowing more targeted recruitment for prospective clinical trials ${ }^{19}$. The majority of these patients have diagnoses, demographics, procedure codes, laboratory results, and medications in HERON. As capabilities of the team develop we plan to expand to other Frontiers affiliated network institutions, state agencies, and our rural practice networks (outlined in aim 4). This may require centralized and/or distributed data integration ${ }^{20,21}$.

Aim \#3: Advance medical innovation by linking biological tissues to clinical phenotype and the pharmacokinetic and pharmacodynamic data generated by research in phase I and II clinical trials (addressing T1 translational research).
We saw two points where Frontiers' informatics must bridge between basic science resources and clinical activities to enhance our region's ability to conduct translational research. Our region provides a wealth of biological and analytical capabilities but struggles at times to understand if our clinical environments see enough patients to support research. This leads to clinical trials which fail to accrue enough subjects ${ }^{22}$. Accurately annotating biological tissues and routine clinical pathology specimens with the patients' phenotypic characteristics (such as diagnosis and medications from the clinical records) would provide a more accurately characterized clinical research capacity. Our second targeted area is designed to support the Drug Discovery and Development activities of Frontiers' Institute for Advancing Medical Innovation's (IAMI) initiative in managing information in phase I and II clinical trials. Molecular biomarker and pharmacokinetics/pharmacodynamics research activities provide high quality analysis, but their results are not easily integrated with other records maintained in clinical research information systems. Reciprocally, if clinical characteristics could be provided with samples in a standardized manner it would allow improved modeling and analysis by the core laboratories. Collaboration between the bioinformatics and biomedical informatics specifically targeted at biospecimens, pharmacokinetic/pharmacodynamic results and clinical trials will provide a foundation for subsequent integration and our final goal of providing molecular bioinformatics methods. 
Aligning CTSA capabilities with our Cancer Center's pursuit of national designation was a top T1 priority for the informatics team. Our ability to incorporate clinical pathology based information and the biological tissue repositories with HERON could improve cohort identification, clinical trial accrual, and clinical trial characterization. Patients' tissue specimens are a fundamental resource that links the majority of basic biological science analysis to clini-

\section{i2b2 Query \& Analysis Tool}

\section{Navigate Terms Find Terms}

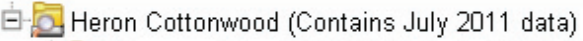

Demographics [15,367,969 facts; $1,858,481$ patients]

+1. Diagnoses [16,413,722 facts; 551,339 patients]

4. Flowsheets [374,860,347 facts]

KUMC BSR Specimens [20,412 facts; 2,247 patients]

al fluid [12,843 facts; 1,936 patients]

+ blood [12,692 facts; 1,933 patients]

+ ather [150 facts; 11 patients]

-

+

- bone, connective tissue, skin, breast [869 facts; 232 patients]

+ digestive systems and peritoneum [1,701 facts; 238 patients]

$\square$ genitourinary [3,733 facts; 849 patients]

D. Bladder [124 facts; 25 patients]

-2] Cervix [33 facts; 10 patients]

D] Endometrium [116 facts; 56 patients]

[.] Fallopian Tube [28 facts; 11 patients]

-5] Kidney [656 facts; 89 patients]

5] Labia [<10 facts]

2] Myometrium [<10 facts]

.2] Ovary [346 facts; 93 patients]

-5] Prostate $[2,293$ facts; 598 patients]

5] Seminal Vesicle [ $<10$ facts]

5] Uterus [119 facts; 23 patients]

5] Vaginal Wall [ $<10$ facts]

De] Vulva [<10 facts]

$\$$ lip, oral cavity, pharynx [301 facts; 45 patients]

\$.

\$- unspecified sites [347 facts; 74 patients]

+ Laboratory Tests [39,974,387 facts; 157,954 patients]

+ Medications [24,366,357 facts; 88,062 patients]

† Procedures [8,861,630 facts; 511,233 patients]

Figure 5. Initial ontology for Biospecimen Tissue Repository Integration within HERON cal relevance. We need to improve our characterization of these samples to maximize our investment in maintaining them for clinical research. By integrating clinical pathology derived characteristics and research specimens with clinical information in HERON, researchers can quantify samples belonging to patients (e.g. aggregating all breast cancer diagnoses using the i2b2 clinical concept "malignant neoplasm of the breast" and treated with the adjuvant therapy Trastuzumab).

On July 12, 2012, KU was awarded NCI Cancer Center Designation, the highest priority strategic objective for the university. Informatics contributed by enhancing $\mathrm{i} 2 \mathrm{~b} 2$ for cancer research by incorporating key data sources and developing methods for conducting real time survival analysis. Our first step towards supporting T1 research was integrating the Biological Tissue Repository (BTR) in the Translational Technologies and Resource Center (Section 8) and HERON. The BTR, built upon the existing KU Cancer Center Biospecimen Shared Resource, plays a vital role in collecting and distributing high quality human biospecimens essential to research. Improving specimen management for clinical research has been identified as a high 
priority initiative for KU. Integrating the BTR allows users to exploit the phenotypic data in HERON to characterize the samples. BTR personnel can also use this linkage to provide investigators requesting samples with clinical phenotype from HERON via a data use request approved by the oversight committee. Figure 5 demonstrates the ontology for biospecimens within HERON's i2b2 application. We are currently migrating the BTR to reside within the Comprehensive Research Information System (CRIS). This will enhance analysis and characterization of data from clinical trials and also improve inventory management, retrieval, and quality assurance processes for the BTR staff.

Our second step was to incorporate the hospital's Tumor Registry within HERON since the validated tumor registry provides additional tumor characteristics for the cancer population, outcomes, and follow up information that are not maintained in surgical pathology

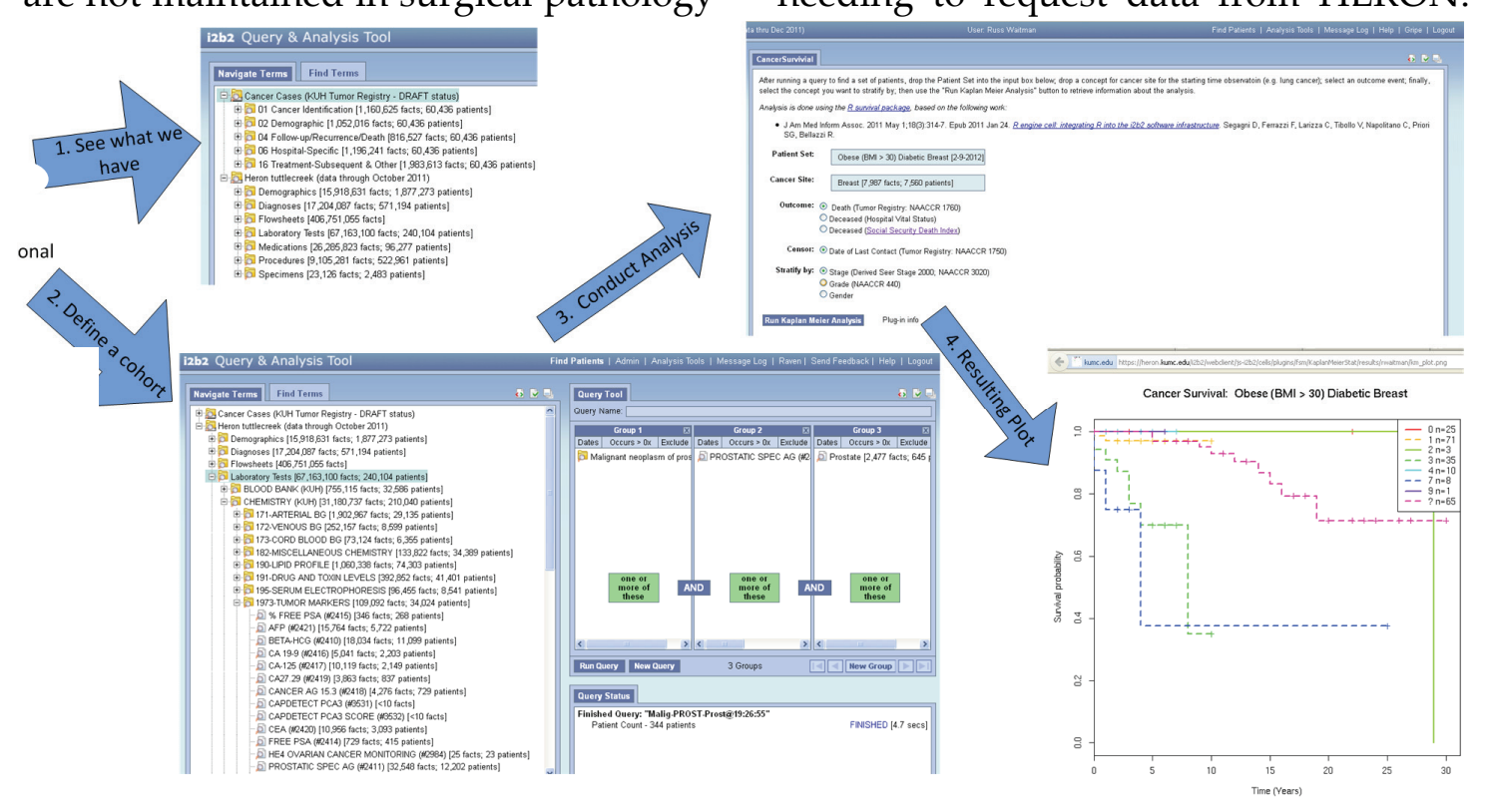

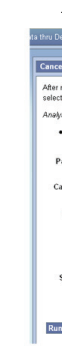

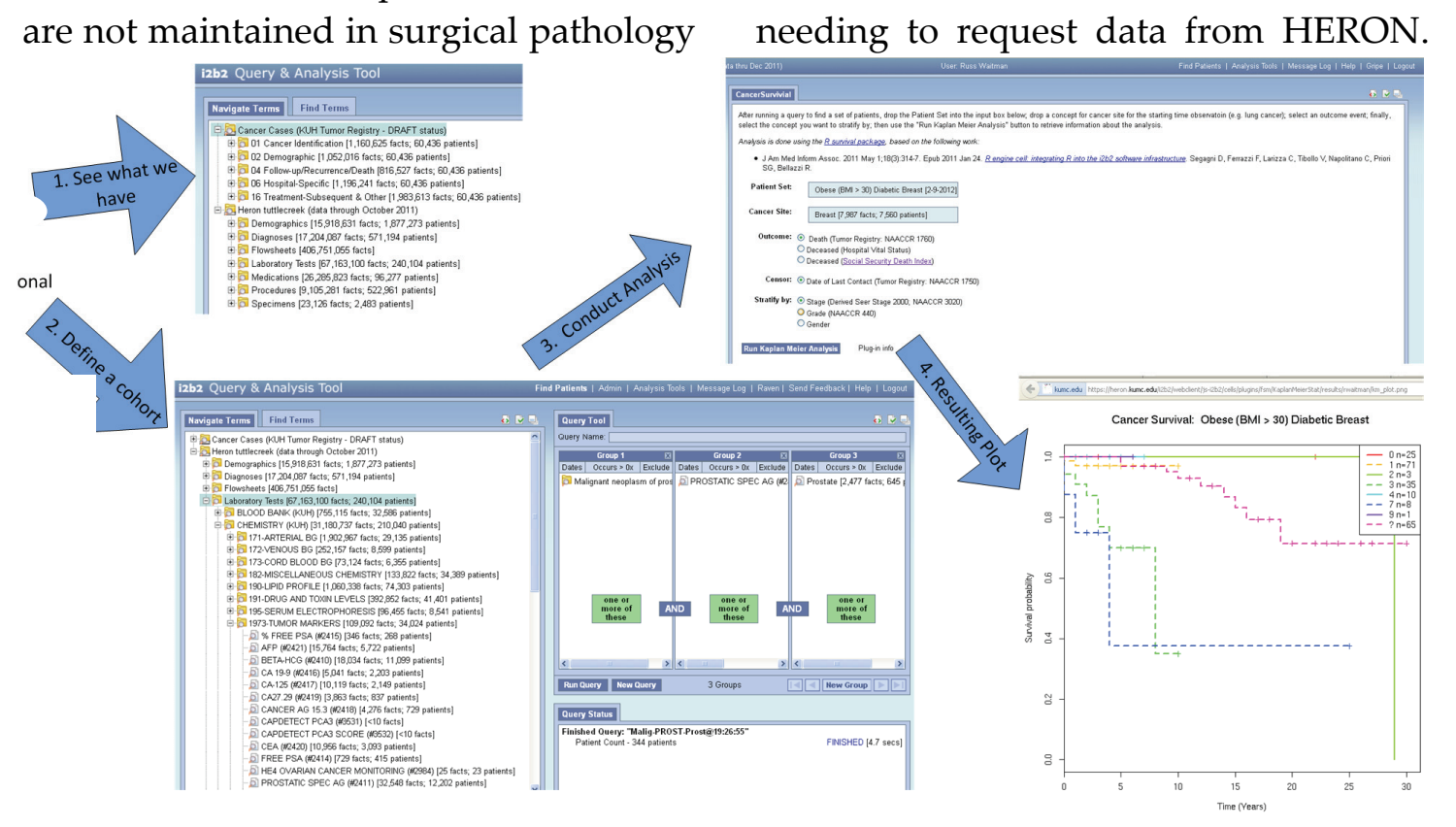
porated with collaboration from Kimmel Cancer Center (PA) and Group Health Cooperative (WA) and leveraged using the national standard for tumor registry data specified by the North American Association for Central Cancer Registries (NAACCR). We also incorporated the social security death index from the National Institutes for Standards and Technology to provide support for long term outcomes and survival analysis across all clinical conditions and health services research. Finally, we created rgate - an improved method to integrate $\mathrm{R}$ with i2b2 and Oracle ${ }^{23}$. Figure 6 provides a summary of HERON workflow for hypotheses generation and preliminary visualization using the interactive analysis tools. Future work will focus on generalizing plugins for multiple cohorts beyond cancer and extending the use of $R$ analysis within HERON so that initial validation can be conducted without needing to request data from HERON.

Figure 6. HERON providing interactive survival analysis 
That in turn reduces the risk of accidental data disclosure.

Going forward, we plan to support IAMI clinical trials by integrating and standardizing information between the bioinformatics and pharmacokinetic/pharmacodynamic (PK/PD) program and CRIS. The Institute for Advancing Medical Innovation (IAMI) builds upon the region's significant drug discovery capabilities and creates a novel platform for medical innovations. Members of the K-INBRE Bioinformatics Core (KBC) provide bioinformatics analysis across the spectrum of target discovery, compound screening, lead identification and optimization, and preclinical development. As drug candidates successfully transition to a new investigational drug application, we will continue bioinformatics support into clinical trials.

Through Frontiers we hope to establish stronger bridges between analytical resources and clinical information which will allow phenotypic variables to be exploited by bioinformatics expertise in data mining by the K-INBRE Bioinformatics Core $(\mathrm{KBC})$ and the Bioinformatics and Computational Life Sciences Research Laboratory. Over time, Frontiers T1 translational research efforts may be enhanced by providing data mining algorithms that extract key information from data-intensive wet lab studies and integrating those results with phenotype derived from clinical applications. This bridge between molecular-level analysis and clinical studies is a potent model for biomarker prediction and validation, PK/PD studies, and the prospective refinement of personalized medicine.
Aim \#4: Leverage an active, engaged statewide telemedicine and Health Information Exchange (HIE) to enable community based translational research (addressing T2 translational research).

In the latter years of the grant we plan to leverage regional informatics capabilities to complement the long history of engaging the community through outreach via telemedicine, continuing medical education, and our extensive community research projects in urban rural and frontier settings. We will highlight the initial areas where Frontiers is deploying informatics in support of $\mathrm{T} 2$ community based research.

Under the leadership of Dr. Helen Connors, the Center for Health Informatics has been instrumental for advancing HIE for Kansas and the Kansas City bistate region. As chair of the eHealth Advisory Council, Dr. Connors guided the process to establish the Kansas HIE (KHIE) ${ }^{23}$; a non-profit corporation incorporated by the governor in response to the federal HITECH Act. Allen Greiner, MD, director of the Community Partnership for Health (Section 5), participates in selecting electronic health records for the Kansas Regional Extension Center and Dr. Russ Waitman has participated in activities on behalf of Kansas Medicaid and technical subcommittees responsible for the development of Health Information Exchange in the state. The shared goal is to keep Frontiers engaged in state and regional infrastructure capabilities for translational research and facilitate identifying other collaborating organizations. 
The University of Kansas Center for Telemedicine and TeleHealth (KUCTT) provides enormous reach into diverse communities for implementing clinical trials and other research across the state. Telemedicine has figured prominently in a number of clinical research programs (e.g. studies of diagnostic accuracy, cost effectiveness, and patient and provider satisfaction with telemedicine) as well as studies that employ telehealth for randomized control trial interventions. Complementing KUCTT, the Midwest Cancer Alliance (MCA) is a membership-based organization bringing cancer research, care and support professionals together to advance the quality and reach of cancer prevention, early detection, treatment, and survivorship in the heartland. The MCA links the University of Kansas Cancer Center with the hospitals, physicians, nurses and patients battling cancer throughout Kansas and Western Missouri. The MCA advances access to cutting-edge clinical trials as well as professional education, networking, and outreach opportunities. These clinical trials use our Comprehensive Research Information System (CRIS). Notably, the MCA critical access and rural referral centers are also the most active participants in telemedicine. The medical director of the MCA, Dr. Gary Doolittle is a pioneer user and has published telemedicine research with Dr. Ryan Spaulding, the director of the telemedicine network ${ }^{24,25}$. The network has been successfully used to facilitate clinical research and to support special needs populations; especially in the area of smoking cessation.
Biomedical informatics works with KUCTT to provide a telemedicine clinical trials network connected by CRIS for remote data collection via study personnel or direct data entry through a patient portal.

Additionally, in March 2011, Kansas City, Kansas "won" a nationwide Request for Information (RFI) solicitation for construction of a next generation broadband network by Google achieving upload and download speeds of 1 gigabit per second (Google 2011). Google subsequently announced the expansion of service to Kansas City, Missouri for a potential deployment of 500,000 individuals. Through Frontiers, the University of Kansas Medical Center's (KUMC) early support led to a collaborative working relationship between KUMC Medical Informatics and the Google Fiber team to explore leveraging the technology to further health care delivery, education, and research. On July 26, 2012, Google unveiled their services and began preregistration throughout the Kansas City metropolitan area. KUMC is actively participating by providing nutritional consults via telepresence as part of the Google Fiber Space located a few blocks from the medical center campus. Building on the strength of telemedicine and informatics Drs. Waitman and Spaulding are collaborating with the School of Nursing and the School of Engineering to develop a research proposal for the National Science Foundation's US Ignite (www.nsf.gov/usignite) initiative to develop next generation applications that can exploit ultra-fast networks such as Google Fiber. 
In the coming year, Frontiers plans to build upon investment in electronic medical records by consulting with Frontiers investigators to optimize the use of clinical systems for disseminating translational evidence and recording measures to verify adoption. This work is also foundational to medical informatics and will allow the evaluation of native clinical information system "signals" against manual processes used to report measures to government, regulatory agencies, and national registries. Working with the hospital to develop capabilities to acquire user activity data to measure the systems' impact on workflow and clinical decision making (e.g. drugdrug interaction overrides, time to complete medication administration task) will also help us overcome translational research's last mile: implementation into clinical workflow.

Longer term objectives for Frontiers will look to develop methods to engage with community providers regarding electronic health record adoption and those systems' capacity to support translational research. As mentioned previously, we have also made initial inroads regarding incorporating state and national data sources into HERON as illustrated by our incorporation of the Social Security Death Index. Over time, we hope to work with the Kansas Medicaid program to investigate methods to link health data maintained by the state as claims against the clinical data in HERON. This will further three objectives: (a) national data may provide outcome measures lacking in acute care clinical information systems, (b) national registries, such as the National Database of Nursing Quality Indicators, can evaluate the degree to which manually ab- stracted measures might be automatically derived from a mature clinical information system, and (c) data integration will allow hypotheses to be explored that suggest methods for improving care. Finally, we aim to collaborate with Frontier's Personalized Medicine and Outcomes Center to provide complex risk models for decision support to a variety of clinical specialties. Those investigators have developed methods that translate complex risk models into fully functional decision-support tools for physicians as well as personalized educational and informed consent documents for patients.

\section{Acknowledgements}

Large portions of this text were previously used in the university CTSA application's biomedical informatics section by the coauthors Lemuel Waitman, Judith Warren and Gerald Lushington. We would like to acknowledge the participation of Frontiers leadership and numerous investigators throughout the Kansas City region who have contributed to the development and ongoing progress of Frontiers.

\section{References}

1. Zerhouni EA. Translational and clinical sciencetime for a new vision. NEJM 2005, 353:1621-23.

2. The University of Kansas Medical Center 2012. Institute for Advancing Medical Innovation. Retrieved from http://www.kumc.edu/iami.html

3. Pulley JM, Harris PA, Yarbrough T, Swafford J, Edwards T, Bernard GR. Acad Med. 2010 Jan;85(1):164-8. An informatics-based tool to assist researchers in initiating research at an academic medical center: Vanderbilt Customized Action Plan.

4. Harris PA, Taylor R, Thielke R, Payne J, Gonzalez N, Conde JG. Research electronic data capture (REDCap) - A metadata-driven methodology and workflow process for providing translational research informatics support, J Biomed Inform. 2009 Apr;42(2):377-81.

5. Sears CS, Prescott VM, McDonald CJ. The Indiana Network for Patient Care: A Case Study of a Successful Healthcare Data Sharing Agreement. 
American Bar Association Health eSource, September 2005.

6. Weber GM, Murphy SN, McMurry AJ, Macfadden D, Nigrin DJ, Churchill S, Kohane IS. The Shared Health Research Information Network (SHRINE): a prototype federated query tool for clinical data repositories. J Am Med Inform Assoc. 2009 Sep-Oct;16(5):624-30.

7. Roden DM, Pulley JM, Basford MA, Bernard GR, Clayton EW, Balser JR, Masys DR. Development of a large-scale de-identified DNA biobank to enable personalized medicine. Clin Pharmacol Ther. 2008 Sep;84(3):362-9. Epub 2008 May 21.

8. Pulley J, Clayton E, Bernard GR, Roden DM, Masys DR. Principles of human subjects protections applied in an opt-out, de-identified biobank. Clin Transl Sci. 2010 Feb;3(1):42-8.

9. Murphy SN, Weber G, Mendis M, Gainer V, Chueh HC, Churchill S, Kohane I. Serving the enterprise and beyond with informatics for integrating biology and the bedside (i2b2). J Am Med Inform Assoc. 2010 Mar-Apr;17(2):124-30.

10. SP 800-66 Rev 1 Oct 2008 An Introductory Resource Guide for Implementing the Health Insurance Portability and Accountability Act (HIPAA) Security Rule. http://csrc.nist.gov/publications/nistpubs/800-66Rev1/SP-800-66-Revision1.pdf

11. Friedlin FJ, McDonald CJ. A software tool for removing patient identifying information from clinical documents. J Am Med Inform Assoc. 2008 Sep-Oct;15(5):601-10. Epub 2008 Jun 25.

12. Loukides G, Denny JC, Malin B. The disclosure of diagnosis codes can breach research participants' privacy. J Am Med Inform Assoc. 2010 May 1;17(3):322-7.

13. Benitez K, Malin B. Evaluating re-identification risks with respect to the HIPAA privacy rule. J Am Med Inform Assoc. 2010 Mar 1;17(2):169-77.

14. Karp DR, Carlin S, Cook-Deegan R, Ford DE, Geller G, Glass DN, Greely H, Guthridge J, Kahn J, Kaslow R, Kraft C, Macqueen K, Malin B, Scheuerman RH, Sugarman J. Ethical and practical issues associated with aggregating databases. PLoS Med. 2008 Sep 23;5(9):e190.

15. Yeniterzi R, Aberdeen J, Bayer S, Wellner B, Hirschman L, Malin B. Effects of personal identifier resynthesis on clinical text de-identification. J Am Med Inform Assoc. 2010 Mar 1;17(2):159-68.

16. Wynden R, Weiner MG, Sim I, Gabriel D, Casale M, Carini S, Hastings S, Ervin D, Tu S, Gennari J, Anderson N, Mobed K, Lakshminarayanan P, Massary M, Cucina R. Ontology mapping and data discovery for the translational investigator. Pre- sented at the 2010 AMIA Summit on Clinical Research Informatics meeting, March 12-13 2010, San Francisco, CA.

17. Waitman LR, Warren JJ, Manos EL, Connolly DW. Expressing observations from electronic medical record flowsheets in an i2b2 based clinical data repository to support research and quality improvement. AMIA Annu Symp Proc. 2011;2011:1454-63.

18. Blumenthal D, Tavenner M. The "Meaningful Use" Regulation for Electronic Health Records. N Engl J Med. 2010 Jul 13.

19. Denton, J., Blackwell K., Waitman, L., Kluding, P., Choudhary, A., Bott, M., Brooks, W., Greiner, A., Jamison, R., Klaus, S., O'Brien-Ladner, A., Latinis, K., Aaronson, L., Burns, J., \& Barohn, R. (2012). P334 Frontiers Research Participant Registry. Translational Science 2012 Meeting, Research Professionals Abstracts, Clinical and Translational Science, 5:2, http://onlinelibrary.wiley.com/doi/10.1111/j.17528062.2012.00398.x/full

20. Diamond CC, Mostashari F, Shirky C. Collecting and sharing data for population health: a new paradigm. Health Aff (Millwood). 2009 MarApr;28(2):454-66.

21. Berman JJ. Zero-check: a zero-knowledge protocol for reconciling patient identities across institutions. Arch Pathol Lab Med. 2004 Mar;128(3):3446.

22. Nass SJ, Moses HL, Mendelsohn. A National Cancer Clinical Trials System for the $21^{\text {st }}$ Century: Reinvigorating the NCI Cooperative Group Program. Committee on Cancer Clinical Trials and the NCI Cooperative Group Program; Institute of Medicine. Washington, DC: National Academies Press, 2010.

23. Connolly DW, Adagarla B, Keighley J, Waitman LR. Integrating R efficiently to allow secure, interactive analysis within a clinical data warehouse. The 8th International R User Conference. Vanderbilt University; Nashville, Tennessee, USA June 12-15, 2012.

24. Spaulding RJ, Russo T, Cook DJ, Doolittle GC. Diffusion theory and telemedicine adoption by Kansas health-care providers: critical factors in telemedicine adoption for improved patient access. J Telemed Telecare. 2005;11 Suppl 1:107-9.

25. Doolittle GC, Spaulding RJ. Defining the needs of a telemedicine service. J Telemed Telecare. 2006;12(6):276-84. 